(2) Open Access Full Text Article

REVIEW

\title{
Importance of balanced diet and physical activity during and after cancer treatment in adolescent patients
}

Margaux J Barnes'

Wendy Demark-

Wahnefried ${ }^{2}$

'Department of Psychology, University of Alabama at Birmingham, ${ }^{2}$ University of Alabama at Birmingham Comprehensive Cancer Center, Birmingham, Alabama, USA
Correspondence: Margaux Barnes Department of Psychology, $\mathrm{CH} 415$, University of Alabama at Birmingham, Birmingham, AL 35233, USA

Tel +l 2059343850

Fax + I 20597561110

Emailmargaux@uab.edu
This article was published in the following Dove Press journal:

Clinical Oncology in Adolescents and Young Adults

12 June 2014

Number of times this article has been viewed

\begin{abstract}
Adolescents diagnosed with cancer are at increased risk for current and future health problems and premature death. As such, it is important to foster the development of healthpromoting behaviors that may ameliorate some of this risk. Specific attention has been given to diet and physical activity, as these are behaviors that can be directly controlled and modified by the survivor. Despite the importance of adequate nutrition and physical activity, a large proportion of adolescents with a history of cancer do not meet recommended guidelines for these health behaviors. The current review summarizes the beneficial effects of diet and physical activity in adolescent cancer patients both during and after treatment, evaluates interventions that have been developed to address these behaviors, and provides recommendations for future strategies on how to improve these behaviors in this population. A structured literature review identified ten empirical articles examining diet and/or physical activity interventions in adolescents with a history of cancer. While several interventions aimed at increasing diet and physical activity in this population have been successful, more research is needed to evaluate long-term maintenance of health behaviors, as well as the impact these behavioral changes have on adolescents as they continue into adulthood. Future interventions should incorporate key elements of adolescent development including individualized and specific intervention components and the incorporation of both peer and family support to increase saliency and long-term commitment.
\end{abstract}

Keywords: oncology, adolescence, health-promoting behaviors

\section{Introduction}

Approximately 13,907 individuals under the age of 20 are diagnosed with cancer each year in the USA. ${ }^{1}$ Over $80 \%$ of these patients will be cured of their cancer based on new and refined treatment protocols that have resulted from the collaborative organization of care across national and international clinical trials. Despite the significant improvement in survival rate, treatment has drawbacks, as its effects are felt both during active treatment and throughout survivorship. During treatment, child and adolescent cancer patients may experience significant effects of medical treatment, including nausea, fatigue, pain, loss of appetite, and decreased health-related quality of life. ${ }^{2,3}$ Following treatment, these survivors continue to be at risk for late effects, such as high risk of early mortality from second cancers, cardiac complications, and pulmonary conditions. ${ }^{4}$ Research indicates that two-thirds of survivors experience at least one physical or psychological late effect following treatment for childhood cancer. ${ }^{5}$ Additionally, long-term childhood cancer survivors are over eight times more likely to die prematurely when compared with age- and sex-matched peers in the general population. ${ }^{4}$ 
Given that children and adolescents diagnosed with cancer are at increased risk for current and future health problems and premature death, it is important to foster the development of health promoting behaviors that may ameliorate some of this risk. Specific attention has been given to diet and physical activity (PA), as these are behaviors that can be directly controlled and modified by the survivor. Consuming a nutritionally balanced diet high in fruits and vegetables and lower in fat, as well as engaging in recommended amounts of PA may shield survivors from some of the late effects related to their cancer diagnosis and treatment. In the general population, balanced nutrition and adequate PA can lower risk for obesity, heart disease, cancer, stroke, and diabetes, as well as promote growth and development during childhood. ${ }^{6}$ Additional specific benefits of PA have been noted in child and adolescent survivors of cancer.

Findings indicate that higher PA is associated with better health-related quality of life across the domains of physical, social, and cognitive functioning in childhood cancer survivors. ${ }^{7}$ Further, two recent exercise intervention trials designed for children and adolescents with cancer found that participation in each of the interventions was associated with improved fitness, muscular strength, and mental health, as well as reduced fatigue. ${ }^{8,9}$

Given the importance of diet and PA to childhood cancer survivors' long-term health and well-being, it is essential to encourage these behaviors as early in development as possible. Adolescence is a particularly crucial time during which these behaviors may have a large impact, both in terms of physical development and the development of lifelong habits related to these health behaviors across the lifespan.

Therefore, given the crucial role of these behaviors during adolescence for those with cancer, the current review summarizes the beneficial effects of diet and PA in adolescent cancer patients both during and after treatment, evaluates the success of interventions that have been developed to address these behaviors, and provides recommendations for future strategies on how to improve these behaviors in this population. Specific attention is given to interventions aimed at adolescents both during active treatment and in the survivorship period.

\section{Importance of diet and PA for adolescents with cancer}

Adolescence is a period of rapid physical growth resulting from the simultaneous release of various growth, thyroid, and sex hormones. ${ }^{10}$ At the most rapid period of growth following childhood, adolescents grow at a rate nearly identical to that of a toddler, during which time bones become harder, muscle development increases, and heart and lungs increase in both size and capacity. ${ }^{11,12}$ To achieve optimal physical growth and development, adolescents require adequate nutrition as well as PA to support muscle development and bone health. ${ }^{13,14}$

When a child or adolescent is diagnosed with cancer, normal growth and development can be undermined as a result of both the cancer and treatment-related toxicity. Not only are adolescents with cancer or childhood cancer survivors in need of adequate nutrition and PA to support typical development, but they are in even greater need of support for their development in the context of their cancer diagnosis and treatment. ${ }^{15}$ Although many associate cancer with weight loss and cachexia, such symptoms are generally confined to later-stage or more advanced disease, and over a third of survivors who are normal weight prior to diagnosis are considered overweight by the end of treatment. ${ }^{16}$ Weight gain and adverse body composition change that favors the accumulation of adipose tissue at the expense of lean body mass is particularly pronounced with treatment for specific brain tumors, acute leukemias, and lymphoma. ${ }^{17,18}$ As a result, child and adolescent cancer survivors exhibit several known risk factors for metabolic syndrome, including enlarged waist circumferences and increased body mass index measures, and high triglycerides and low-density lipoprotein cholesterol compared with their healthy siblings. ${ }^{19}$ Finally, compared with peers with no history of cancer, adolescent and young adult cancer survivors report a significantly higher prevalence of obesity, cardiovascular disease, hypertension, asthma, and poor general physical health, highlighting the importance of lifestyle choices on long-term health and well-being in this population. $^{20}$

Despite the increased need for adequate nutrition and PA, a large proportion of adolescents with a history of cancer do not meet recommended guidelines for these health behaviors. In terms of nutrition, 39\%-94\% of survivors of childhood cancer do not meet national recommendations for dietary intake. ${ }^{21}$ Common deficiencies include calcium, vitamin D, folate, and iron, many of which play an important role in cardiovascular health and bone development. ${ }^{22,23}$ Research also indicates that over half of childhood cancer survivors consume more than $110 \%$ of their estimated energy needs on a daily basis, creating a risk for undue weight gain. ${ }^{23}$ A review conducted by Stolley et al found that childhood cancer survivors of all ages reported largely unhealthy dietary habits, with specific concern for low fruit and vegetable intake, low calcium intake, and high fat intake. ${ }^{24}$ These data, taken together with endocrine and metabolic effects 
secondary to several cancer types and treatment regimens, can greatly increase risk for childhood obesity. ${ }^{23}$ DemarkWahnefried and colleagues reported that younger childhood cancer survivors (ie, those $<18$ years old) were more likely to meet calcium guidelines and eat five or more daily servings of fruits and vegetables compared with childhood cancer survivors who were older (ie, $>18$ years old), thus calling for early interventions that are able to "catch" poor dietary habits before they become ingrained. ${ }^{25}$

Similar to dietary behaviors, a large proportion of childhood cancer survivors do not meet PA recommendations. ${ }^{24,26,27}$ The PA of child and adolescent cancer survivors is generally classified according to Center for Disease Control and Prevention recommendations of at least 60 minutes of moderate to vigorous activity per day at least 5 days per week. ${ }^{28}$ Almost all published data indicate that less than $50 \%$ of childhood cancer survivors meet these PA guidelines. ${ }^{24,27}$ Data also suggest that these survivors are less active than healthy comparison groups. ${ }^{24,27}$ In light of evidence suggesting that child and adolescent cancer survivors take in more than their estimated energy needs, this deficit in PA may compound survivors' risk for obesity, metabolic syndrome, bone-density deficiencies, and cardiovascular problems.

\section{Systematic evaluation of diet and PA interventions}

To better understand the strategies used to target improvements in diet and PA, a systematic search of PubMed, Medline $^{\circledR}$, and PsycINFO ${ }^{\circledR}$ was conducted for studies published prior to October 2013. The search consisted of the subject headings and text words: "childhood cancer" or "pediatric cancer" combined with each of the following: "health behaviors", "lifestyle", "diet", "nutrition”, "PA", “exercise", and "intervention". All search results were limited to English language. A secondary search was conducted by manually reviewing the reference sections of the identified manuscripts. After obtaining all relevant manuscripts, abstracts were screened to ensure applicability to the topic. Manuscripts were excluded if they did not 1) report on an intervention addressing diet and/or PA; 2) include adolescents, defined as children between the ages of 11 and 19 years, either receiving treatment for or survivors of cancer; and 3 ) include a sample size of at least ten childhood cancer survivors and/or patients on active treatment for childhood cancer.

Of the ten interventions targeting adolescent nutrition and PA, one addressed both diet and PA, two addressed diet only, and seven addressed PA only. Interventions were heterogeneous across sample sizes (range $=9-251$ ), ages (range $=3-30$ years of age), diagnoses (acute lymphoblastic leukemia, brain tumor, mixed diagnoses), and targeted points of intervention (on-treatment, immediate post-completion of treatment, long-term survivorship). Specific details on the studies are presented in Table 1. The interventions and associated outcomes within the specified behaviors are addressed following.

\section{Diet}

Dietary behavioral interventions aimed at adolescents are limited. Of the two identified interventions, one dietary intervention aimed to improve bone health in a sample of 75 adolescent and young adult survivors who were one or more years off-treatment. Through a half-day behavioral workshop, the intervention aimed to increase participants' awareness of cancer late-effects; reduce barriers to, and increase perceived benefits of, health-promoting behaviors; and improve self-efficacy to lead a healthy lifestyle with a specific emphasis on promotion of good bone-health habits. ${ }^{29}$ At 1-month follow-up, average milk consumption frequency (intervention $=3.36$, standard deviation $[\mathrm{SD}]=0.72$ versus control $=2.93, \mathrm{SD}=0.88$ ) and past-month calcium supplementation (percent with any calcium supplementation 1-month post-intervention: $82.9 \%$ Intervention group versus $24.1 \%$ control group) were significantly greater in the intervention group compared with in the control group. At 1-month follow-up, the intervention group reported a 53.9\% increase in participants with any calcium supplementation across the previous month compared with baseline, while the control group showed an increase of $2.5 \%$. Neither long-term maintenance of bone-health behavior change nor impact on bone density was evaluated.

The second study to include diet as an outcome consisted of a randomized, controlled psychoeducational intervention that encouraged adolescent survivors to select one target health behavior known to be associated with cancer risk for improvement. No significant changes in survivor-selected health behavior changes related to diet were found following the year-long intervention and, interestingly, survivors in the intervention group showed a nonsignificant downward trend related to improved nutrition compared with survivors receiving standard of care (change score: intervention group $=-0.10$ versus standard of care group $=0.01) .{ }^{30,31}$ However, the findings of this study are complicated by non-validated means of assessing dietary intake and expressed concerns that the intervention was delivered in a clinical setting that may have been distracting, if not anxiety provoking. 


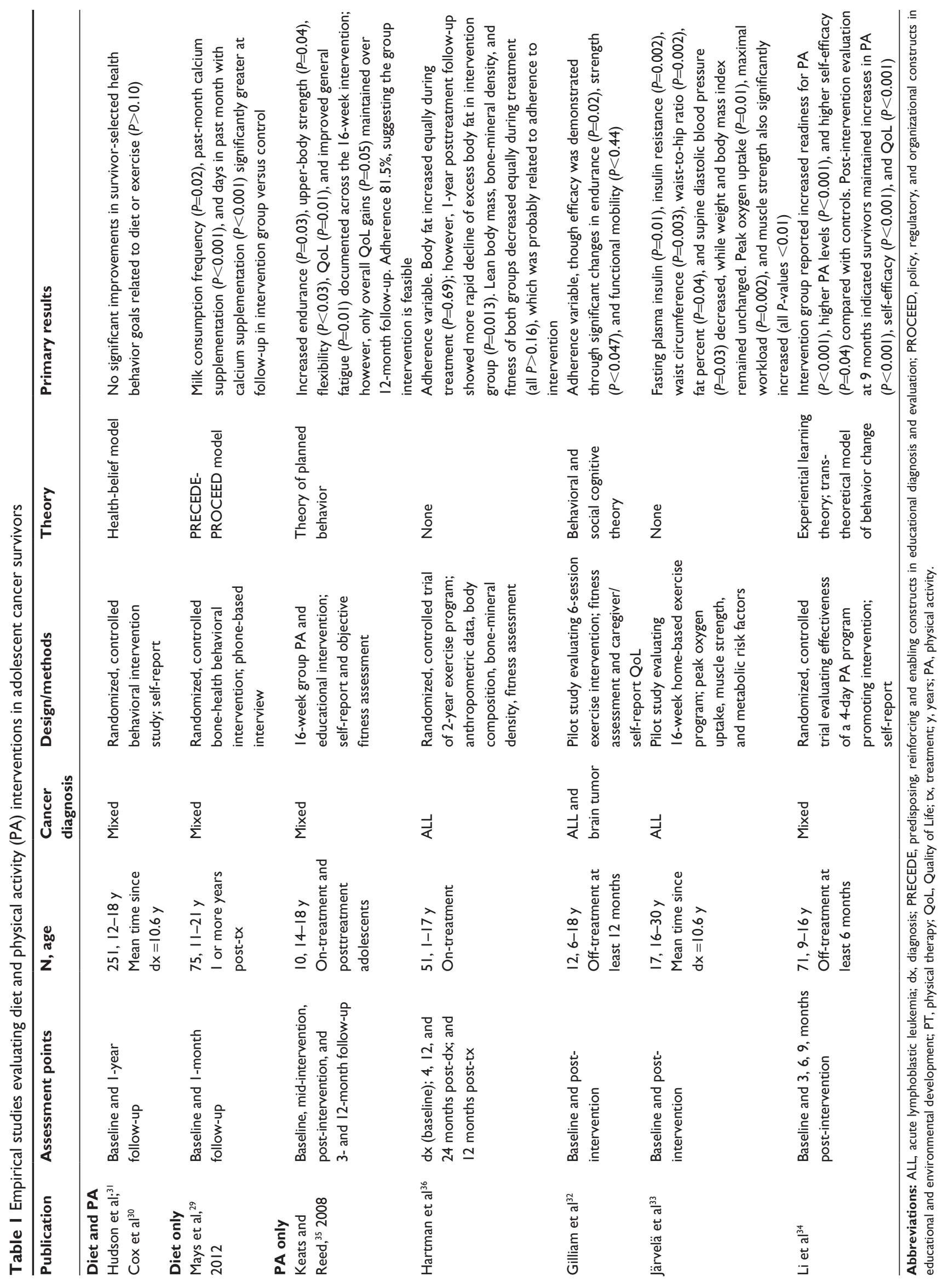




\section{PA}

Comparatively more attention has been given to interventions targeting PA in adolescents with a history of cancer. Of the eight interventions targeting PA, five included survivors only, two included on-treatment participants only, and one included all adolescents diagnosed with cancer regardless of treatment status. Encouragingly, no studies reported negative outcomes for participants in terms of adverse events or perceived risk, either during treatment or during survivorship, suggesting that, from a medical standpoint, PA interventions are safe and appropriate for this population.

For those studies targeting PA in adolescent survivors, four of the six interventions showed significant change in outcomes related to PA following completion of the intervention. ${ }^{32-35}$ Of the studies reporting significant improvements, the majority included both psychoeducational and behavioral training components and involved trained interventionists to ensure participant adherence with the intervention. With the exception of the 4-day, adventure-based, PA-promoting intervention of $\mathrm{Li}$ et al, ${ }^{34}$ successful interventions occurred across multiple weeks (range $=6-16$ weeks) and included a mix of both supervised and unsupervised PA sessions allowing participants to practice learned PA skills at home. Unfortunately, measured outcomes across interventions were variable, with some interventions relying on caregiver- or survivor-reported outcomes and others focusing on objective physiological data. Of the three interventions reporting objective physiological outcomes, results were equivocal for fitness tests including functional mobility, strength, and endurance..$^{32,33,35}$ Of note, one study assessed markers of metabolic syndrome, a known risk in survivors of childhood cancer. ${ }^{33}$ In this 16-week homebased exercise program, results indicated that, immediately following the intervention, fasting plasma insulin $(P=0.01)$, insulin resistance $(P=0.002)$, waist circumference $(P=0.003)$, waist-to-hip ratio $(P=0.002)$, fat percent $(P=0.04)$, and supine diastolic blood pressure decreased $(P=0.03)$. These outcomes were supported by concurrent changes in fitness testing including peak oxygen uptake and maximal workload, which both increased by $5 \%$ ( $P=0.01$ and $P=0.002$, respectively), and muscle strength (sit-up test $[P=0.01]$, back extensor test $[P=0.002]$, full squatting test $[P=0.0004])$. Long-term maintenance of PA and related outcomes were not assessed.

Interventions conducted with adolescents receiving treatment for cancer showed more consistent results, with all three studies reporting significant change on one or more outcomes following the intervention. Keats and Culos-Reed ${ }^{35}$ conducted a 16-week group PA and educational intervention that resulted in increased self-reported PA, improved quality of life, and improved fatigue following completion of the study. ${ }^{35}$ Long-term maintenance of PA and fatigue gains were not sustained, though quality of life remained higher at the 12-month follow-up. Adherence across the 16-week intervention was $81.5 \%$, further supporting the feasibility of PA interventions for adolescents receiving active cancer treatment.

The remaining intervention conducted with adolescents receiving active treatment was a physical therapy intervention implemented at diagnosis for patients with acute lymphoblastic leukemia (ALL) ${ }^{36}$ This intervention consisted of inpatient physical therapy evaluations and sessions during hospital admissions at multiple time points throughout treatment coupled with recommended exercises to be completed by the patient at home. Adherence was variable, which is not surprising given the lengthy time of treatment and significant complications that can occur during treatment for ALL. Participants were evaluated at diagnosis, 32 weeks post-diagnosis, 1 year post-diagnosis, at the cessation of treatment (approximately 2 years post-diagnosis), and 1 year posttreatment. Results indicated that body fat increased equally for all patients during treatment; however, participants who completed the intervention showed a more rapid decline of excess body fat following treatment compared with those receiving standard care $(P=0.013)$. One year following completion of treatment, the percentage of body fat had decreased in the intervention group (change in percent body fat of -1.08 SD scores) to a greater degree than in the control group (change in percent body fat of -0.49 SD scores $[P=0.013])$. This is significant given the potential for excess weight gain during treatment for ALL followed by steady increases in weight through survivorship for some patients. While preliminary, this study suggests that interventions delivered during active therapy may help adolescents maintain or improve PA behaviors during treatment, thus preventing or blunting some of the more common physical deficits known to be associated with these diagnoses.

\section{Recommendations}

While several interventions have had promising results regarding the benefit of nutrition and PA interventions in adolescents with a history of childhood cancer, several issues must be considered. When considering these results in their entirety, it is important to note that the reported outcomes from several studies were limited due to small sample sizes and variable adherence rates. ${ }^{32-35}$ Additionally, the absence of long-term follow-up to assess maintenance of dietary 
and PA changes prevents drawing conclusions as to the intervention strategies and components necessary to improve durable changes in these behaviors. Specifically, only two studies evaluated long-term maintenance of PA behavior changes and the results were mixed. ${ }^{34,35}$ Self-reported PA, self-efficacy, and quality of life in the Li et al study ${ }^{34}$ and self-reported quality of life alone in the Keats and Culos-Reed study $^{35}$ were maintained at 9 and 12 months, respectively. However, objective increases in physical fitness and/or concurrent fitness levels were not maintained, suggesting that interventions may need to modify or include additional components to increase long-term maintenance. Moreover, the discrepancy in findings also points to a need for the inclusion of objective measures during all time points.

Despite the lack of conclusive evidence to direct the development of interventions aimed at increasing diet and PA in adolescents with cancer, several recommendations are offered here for implementation and evaluation in future studies. While successful interventions include both educational and behavioral components and afford adolescents opportunities for skill development with trained professionals as well as at home or in the community, specific recommendations related to adolescent development may compliment these supported elements. First, the presentation and delivery of key intervention components should be tailored to the developmental stage associated with adolescence. During adolescence, individuals move from concrete thinking patterns to more abstract, logical thought processes while still being primarily self-focused, especially during early adolescence. Following this, engaging an adolescent in the development of his or her own individualized PA and nutrition plan could increase both the saliency of the intervention as well as the individual's motivation and commitment to change. Thus, strategies may include the following: providing a rationale for why lifestyle changes are indicated, taking into account diagnoses and treatment information; defining change goals that are important to the adolescent and what changes they are willing to make; and, finally, providing guidance as to the specific ways in which they could implement these changes into their daily lifestyle given their current familial, social, and environmental context.

Additionally, as adolescents are beginning to form their own self-identity and associate more with their peers as opposed to their parents, the incorporation of peer social support in interventions may be important in addition to parental support. Research indicates that both family and peer support are unique predictors of adolescent cancer survivors' PA levels. ${ }^{37}$ Interventions that incorporate peer support for adolescents may improve the outcomes of interventions on two levels - first, by increasing the direct impact of the intervention on the survivor through peer encouragement for and engagement in diet and PA changes, and, second, via ongoing accountability and social support after the intervention for continued engagement in a healthy lifestyle. Particularly salient to adolescents may be the engagement of peers through current technological means, including social media or smartphone applications that could allow for instantaneous and ongoing peer support. Moreover, these interventions overcome barriers of travel and distance, which are significant for this patient population.

Finally, the timing of the intervention should be considered. While evidence exists in support of interventions delivered at any time point in the cancer journey, the most effective interventions may be those aimed at adolescents prior to the onset of deficits or noted declines in key health behaviors. For this population, interventions introduced at diagnosis or shortly thereafter and incorporated as a standard of care for any child or adolescent undergoing treatment for cancer could prevent or at least mitigate the adoption of poor behaviors and subsequent late effects. Since over $90 \%$ of children and adolescents diagnosed with cancer in the USA are treated at Children's Oncology Group (COG) affiliated institutions, offering these interventions within the context of the COG could also serve as a uniform means of dissemination. ${ }^{38}$ Because of this collaborative and systematic system of care for children and adolescents diagnosed with cancer, it is foreseeable that comprehensive health behavior interventions could be delivered in conjunction with standardized treatment protocols, thus reaching the majority of children and adolescents with cancer. Similar to the refinement of treatment protocols within COG member institutions, behavioral interventions could benefit from collaborative data sharing, allowing for ongoing development, evaluation, and refinement of these interventions, especially with regard to timing, dosing, and delivery.

\section{Conclusion}

Diet and PA play a vital role in the development of adolescents with cancer or those who have been treated for cancer during their youth. Educating survivors on the importance of these behaviors, both for their typical development and in the context of their cancer diagnosis and treatment, is important and may help prevent both acute and late effects related to the cancer journey. While several interventions aimed at increasing diet and PA in this population have been successful, more research is needed to evaluate the long-term maintenance of health behaviors, as well as the impact these 
behavioral changes have on adolescents as they continue into adulthood. Future interventions should incorporate key elements of adolescent development, including individualized and specific intervention components, and peer and family support, to increase saliency and long-term commitment. Finally, collaborative efforts in the development, implementation, and evaluation of these interventions should be coordinated within the childhood cancer professional community to ensure that the greatest numbers of patients are reached with the highest impact possible.

\section{Disclosure}

This work has been supported in part by the National Cancer Institute-sponsored Cancer Prevention and Control Training Program (R25 CA047888). Ms Barnes and Dr DemarkWahnefried have no financial interests or potential conflicts of interest to report in relation to this work.

\section{References}

1. National Cancer Institute (NCI). State cancer profiles [web page on the Internet]. Bethesda, MD: NCI; nd. Available from: http:// statecancerprofiles.cancer.gov/cgi-bin/quickprofiles/profile.pl?00\&515. Accessed February 12, 2014.

2. Eiser C, Eiser JR, Stride CB. Quality of life in children newly diagnosed with cancer and their mothers. Health Qual Life Outcomes. 2005;3:29

3. Penn A, Lowis SP, Stevens MC, et al. Family, demographic and illness-related determinants of HRQL in children with brain tumours in the first year after diagnosis. Pediatr Blood Cancer. 2009;53(6): 1092-1099.

4. Mertens AC, Lui Q, Neglia JP, et al. Cause-specific late mortality among 5 -year survivors of childhood cancer: the Childhood Cancer Survivor Study. J Natl Cancer Inst. 2008;100(19):1368-1379.

5. Hewitt M, Weiner SL, Simone JV, editors; National Cancer Policy Board; Institute of Medicine (IOM); National Research Council. Childhood Cancer Survivorship: Improving Care and Quality of Life. Washington DC: National Academics Press; 2003.

6. Agricultural Research Service, US Department of Agriculture, US Department of Health and Human Services. Report of the Dietary Guidelines Advisory Committee on the Dietary Guidelines for Americans, 2010: to the Secretary of Agriculture and the Secretary of Health and Human Services. Washington DC: US Department of Agriculture; 2010. Available from: www.cnpp.usda.gov/publications/ dietaryguidelines/2010/dgac/report/2010dgacreport-camera-readyjan11-11.pdf. Accessed April 16, 2014.

7. Paxton RJ, Jones LW, Rosoff PM, Bonner M, Ater JL, DemarkWahnefried W. Associations between leisure-time physical activity and health-related quality of life among adolescent and adult survivors of childhood cancers. Psychooncology. 2010;19(9):997-1003.

8. Keats MR, Culos-Reed N. A theory-driven approach to encourage physical activity in pediatric cancer survivors: a pilot study. $J$ Sport Exerc Psychol. 2009;31(2):267-283.

9. San Juan AF, Fleck SJ, Chamorro-Viña C, et al. Effects of an intrahospital exercise program intervention for children with leukemia. Med Sci Sports Exercise. 2007;39(1):13-21.

10. Steinberg L. Adolescence. 8th ed. New York, NY: McGraw-Hill; 2008.

11. Peterson A, Taylor B. The biological approach to adolescence: biological change and psychological adaptation. In: Adelson J, editor. Handbook of Adolescent Psychology. New York, NY: Wiley; 1980:117-155.
12. Susman EJ, Rogol A. Puberty and psychological development. In: Lerner RM, Steinberg L, editors. Handbook of Adolescent Psychology. 2nd ed. New York, NY: Wiley; 2004:15-44.

13. Sallis JF, Patrick K. Physical activity guidelines for adolescents: consensus statement. Pediatr Exerc Sci. 1994;6(4):302-314.

14. Story M. Nutritional requirements during adolescence. In: McAnarney ER, Kreipe RE, Orr DE, Comerci GD, editors. Textbook of Adolescent Medicine. Philadelphia, PA: WB Saunders; 1992:75-84.

15. Bechard LJ, Duggan C. Cancer treatment. In: Walker WA, Watkins JB, Duggan C, editors. Nutrition in Pediatrics: Basic Science, Clinical Applications. 4th ed. Hamilton, Ontario: BC Decker; 2008: 607-616.

16. Love E, Schneiderman JE, Stephens D, et al. A cross-sectional study of overweight in pediatric survivors of acute lymphoblastic leukemia (ALL). Pediatr Blood Cancer. 2011;57(7):1204-1209.

17. Fuemmeler BF, Pendzich MK, Clark K, et al. Diet, physical activity, and body composition changes during the first year of treatment for childhood acute leukemia and lymphoma. J Pediatr Hematol Oncol. 2013;35(6):437-443.

18. Lek N, Prentice P, Williams RM, Ong KK, Burke GA, Acerini CL. Risk factors for obesity in childhood survivors of suprasellar brain tumours: a retrospective study. Acta Paediatr. 2010;99(10):1522-1526.

19. Steinberger J, Sinaiko AR, Kelly AS, et al. Cardiovascular risk and insulin resistance in childhood cancer survivors. $J$ Pediatrics. 2012;160(3):494-499.

20. Tai E, Buchanan N, Townsend J, Fairley T, Moore A, Richardson LC. Health status of adolescent and young adult cancer survivors. Cancer. 2012;118(19):4884-4890.

21. Badr H, Chandra J, Paxton R, et al. Health-related quality of life, lifestyle behaviors, and intervention preferences of survivors of childhood cancer. J Cancer Surviv. 2013;7(4):523-534.

22. Bilariki K, Anagnostou E, Masse V, et al. Low bone mineral density and high incidences of fractures and vitamin D deficiency in 52 pediatric cancer survivors. Horm Res Pediatric. 2010;74(5):319-327.

23. Cohen J, Wakefield CE, Fleming CA, Gawthorne R, Tapsell LC, Cohn RJ. Dietary intake after treatment in child cancer survivors. Pediatr Blood Cancer. 2012;58(5):752-757.

24. Stolley MR, Restrepo J, Sharp LK. Diet and physical activity in childhood cancer survivors: a review of the literature. Ann Behav Med. 2010;39(3):232-249.

25. Demark-Wahnefried W, Werner C, Clipp EC, et al. Survivors of childhood cancer and their guardians. Cancer. 2005;103(10):2171-2180.

26. San Juan AF, Wolin K, Lucía A. Physical activity and pediatric cancer survivorship. Recent Results Cancer Res. 2011;186:319-347.

27. Winter C, Müller C, Hoffmann C, Boos J, Rosenbaum D. Physical activity and childhood cancer. Pediatr Blood Cancer. 2010;54(4):501-510.

28. Haskell WL, Lee I, Pate RR, et al. Physical activity and public health: updated recommendation for adults from the American College of Sports Medicine and the American Heart Association. Med Sci Sport Exer. 2007;39(8):1423-1434.

29. Mays D, Gerfen E, Mosher RB, Shad AT, Tercyak KP. Validation of a milk consumption stage of change algorithm among adolescent survivors of childhood cancer. $J$ Nutr Educ Behav. 2012;44(5):464-468.

30. Cox CL, McLaughlin RA, Rai SN, Steen BD, Hudson MM. Adolescent survivors: a secondary analysis of a clinical trial targeting behavior change. Pediatr Blood Cancer. 2005;45(2):144-154.

31. Hudson MM, Tyc VL, Srivastava DK, et al. Multi-component behavioral intervention to promote health protective behaviors in childhood cancer survivors: the protect study. Med Pediatr Oncol. 2002;39(1):2-10; discussion 2.

32. Gilliam MB, Ross K, Walsh A, et al. Healthy Heroes: a token economy to increase adherence with a community-based exercise intervention for childhood cancer survivors. Rehab Oncol. 2011;29:16-22.

33. Järvelä LS, Kemppainen J, Niinikoski H, et al. Effects of a home-based exercise program on metabolic risk factors and fitness in long-term survivors of childhood acute lymphoblastic leukemia. Pediatr Blood Cancer. 2012;59(1):155-160. 
34. Li W, Chung OKJ, Ho KY, Chiu SY, Lopez V. Effectiveness of an integrated adventure-based training and health education program in promoting regular physical activity among childhood cancer survivors. Psychooncology. 2013;22(11):2601-2610.

35. Keats MR, Culos-Reed SN. A community-based physical activity program for adolescents with cancer (project TREK): program feasibility and preliminary findings. J Pediatr Hematol Oncol. 2008;30(4): 272-280.

36. Hartman A, te Winkel ML, van Beek RD, et al. A randomized trial investigating an exercise program to prevent reduction of bone mineral density and impairment of motor performance during treatment for childhood acute lymphoblastic leukemia. Pediatr Blood Cancer. 2009;53(1):64-71.
37. Gilliam MB, Madan-Swain A, Whelan K, Tucker DC, DemarkWahnefried W, Schwebel DC. Social, demographic, and medical influences on physical activity in child and adolescent cancer survivors. J Pediatr Psychol. 2012;37(22):198-208.

38. Children's Oncology Group. About us [web page on the Internet]. Monrovia, CA: Children's Oncology Group. Available from: http:// www.childrensoncologygroup.org/index.php/about-us. Accessed January 24, 2014.
Clinical Oncology in Adolescents and Young Adults

\section{Publish your work in this journal}

Clinical Oncology in Adolescents and Young Adults is an international, peer-reviewed, open access journal publishing original research, reports, editorials, reviews and commentaries on all aspects of epidemiology, diagnosis and treatment of cancers in adolescents and young adults. The manuscript management system is completely

\section{Dovepress}

online and includes a very quick and fair peer-review system. Visit http://www.dovepress.com/testimonials.php to read real quotes from published authors.

\footnotetext{
Submit your manuscript here: http://www.dovepress.com/clinical-oncology-in-adolescents-and-young-adults-journal
} 